\title{
Effect of Environmental Parameters on the Performance of Optical Wireless Communications
}

\author{
Hosam Abd Elrazek Mohamed Ali $\mathbb{D}^{1,2}$ \\ El-Sayed Soliman A. Said, ${ }^{2}$ and Mohamed Ebrahim Yousef ${ }^{2}$ \\ ${ }^{1}$ Telecom Egypt, Egypt \\ ${ }^{2}$ Al-Azhar University, Egypt \\ Correspondence should be addressed to Hosam Abd Elrazek Mohamed Ali; hosam.ali@te.eg
}

Received 27 November 2018; Revised 25 February 2019; Accepted 25 March 2019; Published 2 May 2019

Guest Editor: Surinder Singh

Copyright (C) 2019 Hosam Abd Elrazek Mohamed Ali et al. This is an open access article distributed under the Creative Commons Attribution License, which permits unrestricted use, distribution, and reproduction in any medium, provided the original work is properly cited.

\begin{abstract}
Free space optical communication systems are the future of the communication systems which can meet the needs of the end users who are demanding high bandwidths to support high-speed communication. These merits are due to its numerous advantages as well as their better performance. This paper studies the effect of environmental parameters like rain, fog, haze, snow, and dust on the performance of optical wireless communications using Opti-system program. This work considers the effect of visibility as well as operating wavelengths on atmospheric attenuation in different weather conditions for free space optical link. For different weather conditions the max distance between transmitter and receiver can also be estimated. The system analysis depicted the better performance of the Multi-Input/Multi-Output (MIMO) configurations compared to Single Input/Single Output (SISO) at different weather conditions.
\end{abstract}

\section{Introduction}

Optical wireless communication is a concept of transmitting information through air with light as a carrier wave [1]. The free space communication is an effective technique with many special advantages such as no need for physical connection between transmitter and receiver. This technique is also easy to install and there are no dangers of radiation where the line of sight is normally allocated in a safe region. Moreover, it shows low power consumption and low bit error rate (BER). Free space optical communication is a line of sight wireless technology that could be deployed in applications where there is a need for a high-speed connectivity. The concept is basically the same as the fiber optic transmissions except for the channel being the free space. High data rates of FSO communication systems have attracted much attention in recent years, thanks to their merits including huge data rates, license-free spectrum, low power consumption, and immunity to the electromagnetic interference. Free space optics link offers gigabit per second data rates with less system complexity. The free space optics system has come up as a better alternative to radio frequency technology [2]. From past decade FSO has gained more importance in both indoor and outdoor applications. There are other applications of FSO, which are to create a Metropolitan Area Networks (MAN), Local Area Networks (LAN) connectivity, optical fiber communication backup, and backhaul for wireless cellular networks [3]. In spite of these advantages, the quality of FSO links is highly depending on the atmospheric weather conditions. The most important phenomena that affect the performance of a FSO link are absorption, scattering, and atmospheric turbulence [4]. Surveying the literature review of this subject is clearly displaying that, in spite of the advantages of these systems, they have individually studied the weather conditions such as fog, haze, snow, and dust. In [5] the effects of temperature on the performances of FSO transmission under Qatar's climate are studied at wavelength $=1550 \mathrm{~nm}$ using two FSO transceivers $\left(2 \mathrm{~T}_{\mathrm{X}} / 2 \mathrm{R}_{\mathrm{X}}\right)$. In [6] studying atmospheric dust effect on the performance of the system leads to conclusion that the visibility decreases with the increase of 


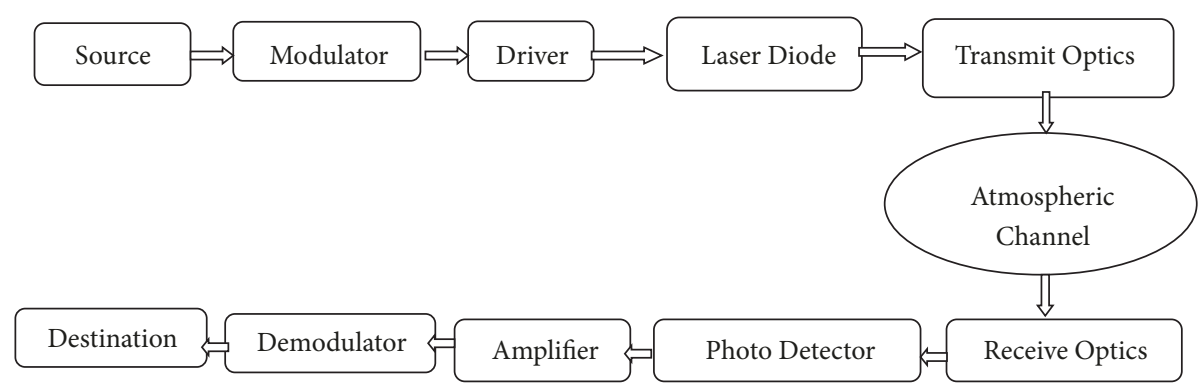

FIGURE 1: Schematic diagram of free space optics.

concentration of dust when the wavelength is $1550 \mathrm{~nm}$ using $1 \mathrm{~T}_{\mathrm{X}} / 1 \mathrm{R}_{\mathrm{X}}$. The haze and fog state effects on the performance of free space optical communications system in Malaysia at 1550 $\mathrm{nm}$ are studied clearly in [7]. The rain, fog, and scintillation state effects are studied clearly in [8] at wavelength $=1310 \mathrm{~nm}$. The haze and fog state effects are studied clearly in $[9,10]$. The rain and fog state effects are studied clearly in [11, 12]. This paper studies the effect of environmental parameters such as rain, fog, haze, snow, and dust on the performance of optical wireless communications using different channels $\left(1 \mathrm{~T}_{\mathrm{X}} / 1 \mathrm{R}_{\mathrm{X}}\right.$, $2 \mathrm{TX} / 2 \mathrm{R}_{\mathrm{X}}$, and $\left.4 \mathrm{~T}_{\mathrm{X}} / 4 \mathrm{R}_{\mathrm{X}}\right)$ at different wavelengths $(850 \mathrm{~nm}$ $1550 \mathrm{~nm})$.

\section{Environmental Attenuations}

Fog is the most hostile factor to FSO link due to the fog particles which are having nearly the same wavelength as wavelength of light. The atmospheric attenuation coefficient is calculated depending on visibility and the wavelength of the incident beam according to [9]

$$
\alpha=\frac{3.91}{v}\left(\frac{\lambda}{\lambda_{0}}\right)^{-q}
$$

where $\lambda$ indicates transmission wavelength $(\mathrm{nm}), \mathrm{V}$ indicates visibility $(\mathrm{km}), \lambda_{0}$ indicates visibility reference wavelength $=550 \mathrm{~nm}$, and $\mathrm{q}$ indicates the size distribution of the scattering particles.

According to Kruze model ( $q=1.6$ if $v>50 \mathrm{~km}, q=1.3$ if 50 $\mathrm{km}>v>6 \mathrm{~km}$, and $q=0.585 v^{1 / 3}+0.34$ if $\left.v<6 \mathrm{~km}\right)$, the attenuation of the transmitted signal $(\alpha)$ can be determined by using (1) for different weather conditions. The atmospheric attenuation $(\tau)$ in $\mathrm{dB}$ can be calculated as follows [13]:

$$
\tau=4.3429 * \alpha * L
$$

where $\mathrm{L}$ is distance between transmitter and receiver.

Rain is one of the factors for inducing attenuation in a FSO system. Rain has less impact than fog because wavelength of optical signal is very small as compared to rain drop. And attenuation of rain can be calculated as follows [14]:

$$
A_{\text {rain }}=1.076 * R^{0.67}(\mathrm{db} / \mathrm{km})
$$

where $\mathrm{R}$ is the rain rate in $\mathrm{mm} / \mathrm{h}$.

\section{System Description}

The system block diagram shown in Figure 1 [8] is composed of three main parts: a transmitter system, a receiver system, and communication channel. It can be classified according to the number of transceivers FSO used as in Figure 1.

3.1. SISO FSO. The transmitter system is composed of four subsystems. The first subsystem is the Pseudo-Random Binary Sequence (PRBS) generator. The second subsystem is the Non-Return-to-Zero (NRZ) encoder that encrypts information. The third subsystem is the optical transmitter (laser generator). The fourth subsystem is the Mach-Zehnder modulator that modulates the intensity of the light source according to the output pulse generator. The communication channel between the transmitter and the receiver is the free space. The optical receiver is composed of an avalanche photodiode (APD), optical amplifiers, the filter (Law Pass Bessel Filter) to filter the high frequency of unwanted signals, and $3 \mathrm{R}$ regenerator to determine the electrical signal embedded. The receiver devices are direct detection based devices which directly detect the instantaneous power of the collected optical field as it arrives at the receivers [15]. Figure 2 shows simulation layout of $\left(1 \mathrm{~T}_{\mathrm{X}} / 1 \mathrm{R}_{\mathrm{X}}\right)$ FSO system.

3.2. MIMO FSO Using $2 T_{X} / 2 R_{X}$. An optical transmitter is connected to a fork, which is a component used to duplicate the number of output ports so that each of the signals coming out from fork's output has the same value with the output signal from the previous component connected to it. The first fork produces the multiple beam and this fork is connected to another set of forks which also produces multiple laser beams for better performance at the $\mathrm{o} / \mathrm{p}$ instead of using multiple $\mathrm{cw}$ laser source for a system; we can simply use fork for getting the same effect. These laser beams are combined by the power combiner. At the receiver side power coming from the FSO channels is again combined by power combiner and then fed to the optical receiver as shown in Figure 3. BER analyzer automatically calculates the bit error rate (BER) and shows the eye diagram [16].

3.3. MIMO FSO Using $4 T_{X} / 4 R_{X}$. As before but here we use $4 \mathrm{~T}_{\mathrm{X}} / 4 \mathrm{R}_{\mathrm{X}}$ instead of $2 \mathrm{~T}_{\mathrm{X}} / 2 \mathrm{R}_{\mathrm{X}}$. MIMO take benefit from the spatial diversity and receive multiple independent copies of the same signal at the receiver, hence resulting in increased 


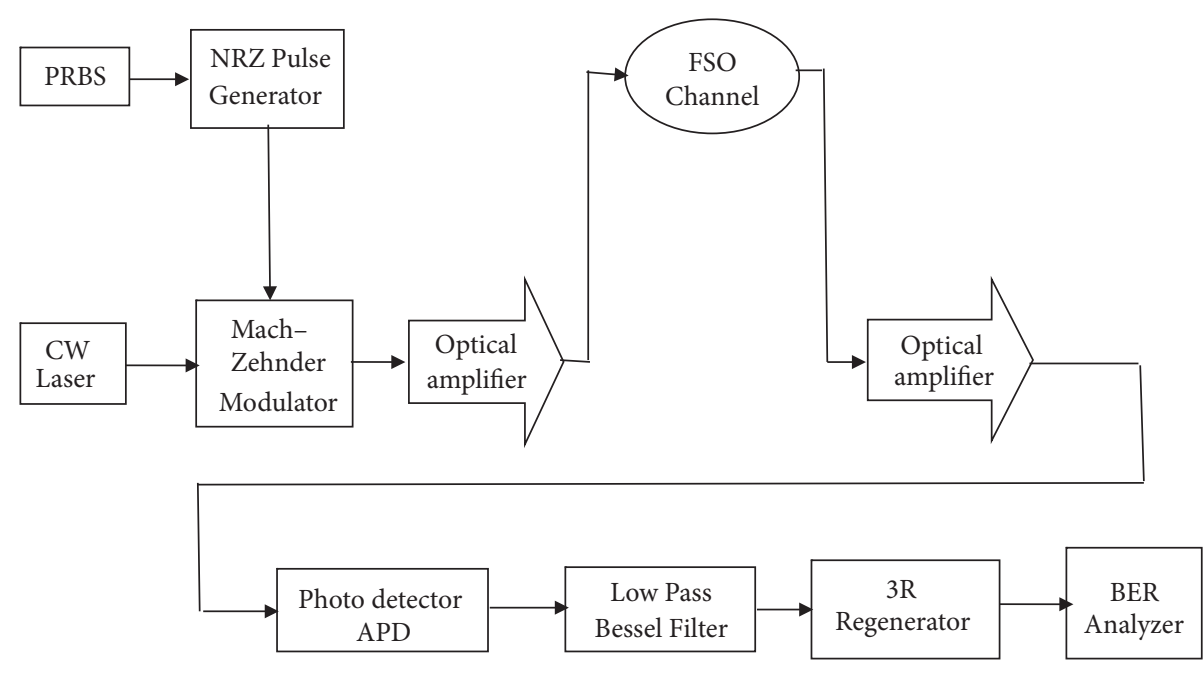

FIgURE 2: Simulation layout of $\left(1 \mathrm{~T}_{\mathrm{X}} / 1 \mathrm{R}_{\mathrm{X}}\right) \mathrm{FSO}$ system.

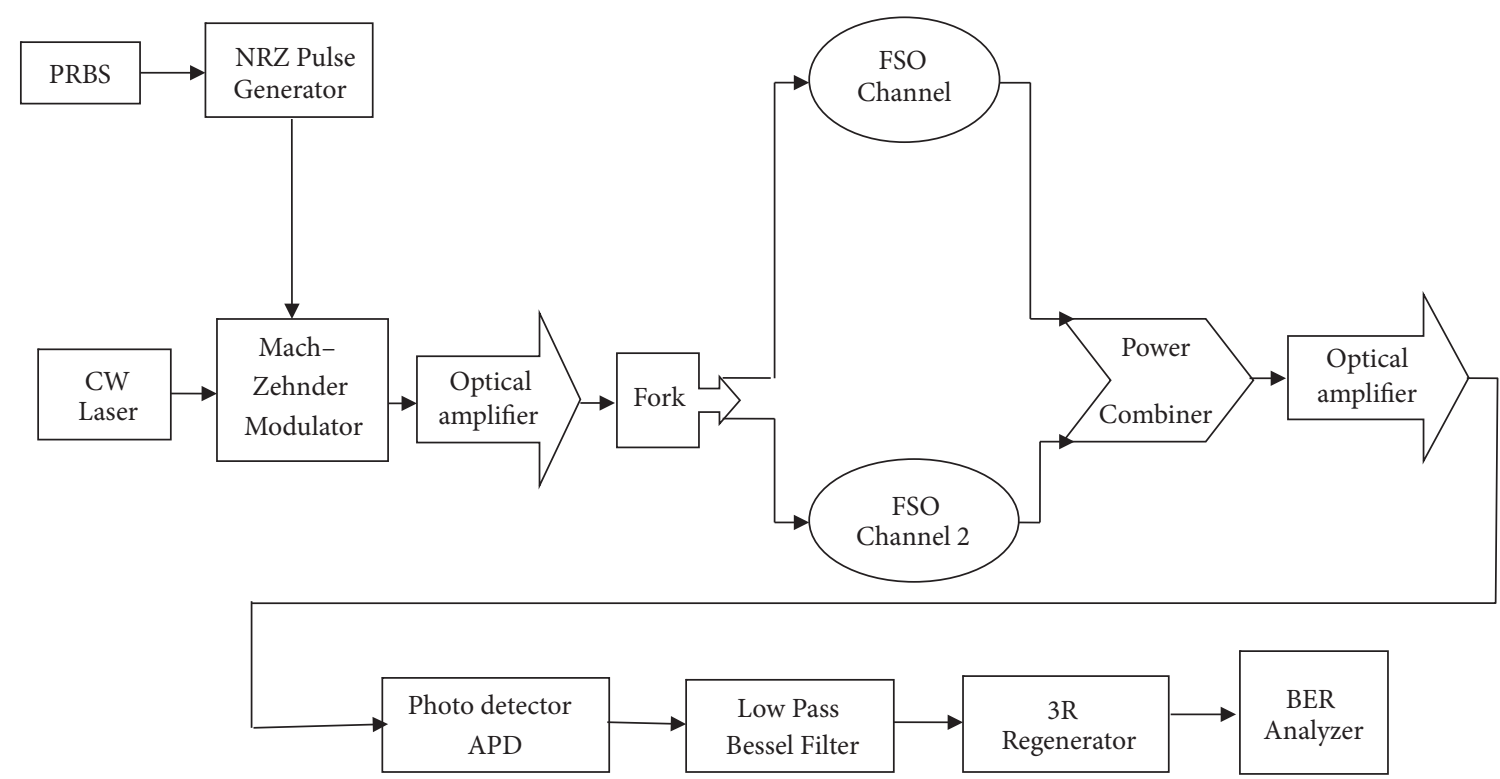

FIgURE 3: Simulation layout of $\left(2 \mathrm{~T}_{\mathrm{X}} / 2 \mathrm{R}_{\mathrm{X}}\right)$ FSO system.

signal to noise ratio. The MIMO technology cannot only increase the data rate but also improve the system reliability through spatial diversity [10]. Figure 4 shows the system using $4 \mathrm{~T}_{\mathrm{X}} / 4 \mathrm{R}_{\mathrm{X}}$.

\section{Simulation Results and Performance Analysis}

This section presents results of Max Q factor, Min BER, and estimated range between transmitter and receiver (three different distances) for different weather conditions using $1 \mathrm{~T}_{\mathrm{X}} / 1 \mathrm{R}_{\mathrm{X}}, 2 \mathrm{~T}_{\mathrm{X}} / 2 \mathrm{R}_{\mathrm{X}}$, and $4 \mathrm{~T}_{\mathrm{X}} / 4 \mathrm{R}_{\mathrm{X}}$ at different wavelengths $(850 \mathrm{~nm}-1550 \mathrm{~nm})$ using Kruze model. The global parameters used in this section are shown in Table 1.
The FSO link medium transmission is the atmosphere that has attenuation depending upon several conditions. Weather conditions are the main cause of attenuation [17]. As observed from (2), atmospheric attenuation of the FSO link is a contributing factor for received power [18]. The most widely used wavelength in the optical communications is allocated in the range of $850 \mathrm{~nm}-1550 \mathrm{~nm}$. Specifically, the wavelength $1550 \mathrm{~nm}$ is the dominantly suitable; due to more than one reason such as it can cover wide distance, deal with high data rate, and grant the eye safety, it can reduce the solar background and finally the light scattering in case of haze/fog.

One can notice that when increasing the wavelength of the transmitted signal, the attenuation would be decreased.

At clear air it can reach a distance of $94 \mathrm{~km}$, while at heavy fog the maximum range is around $0.345 \mathrm{~km}$. 


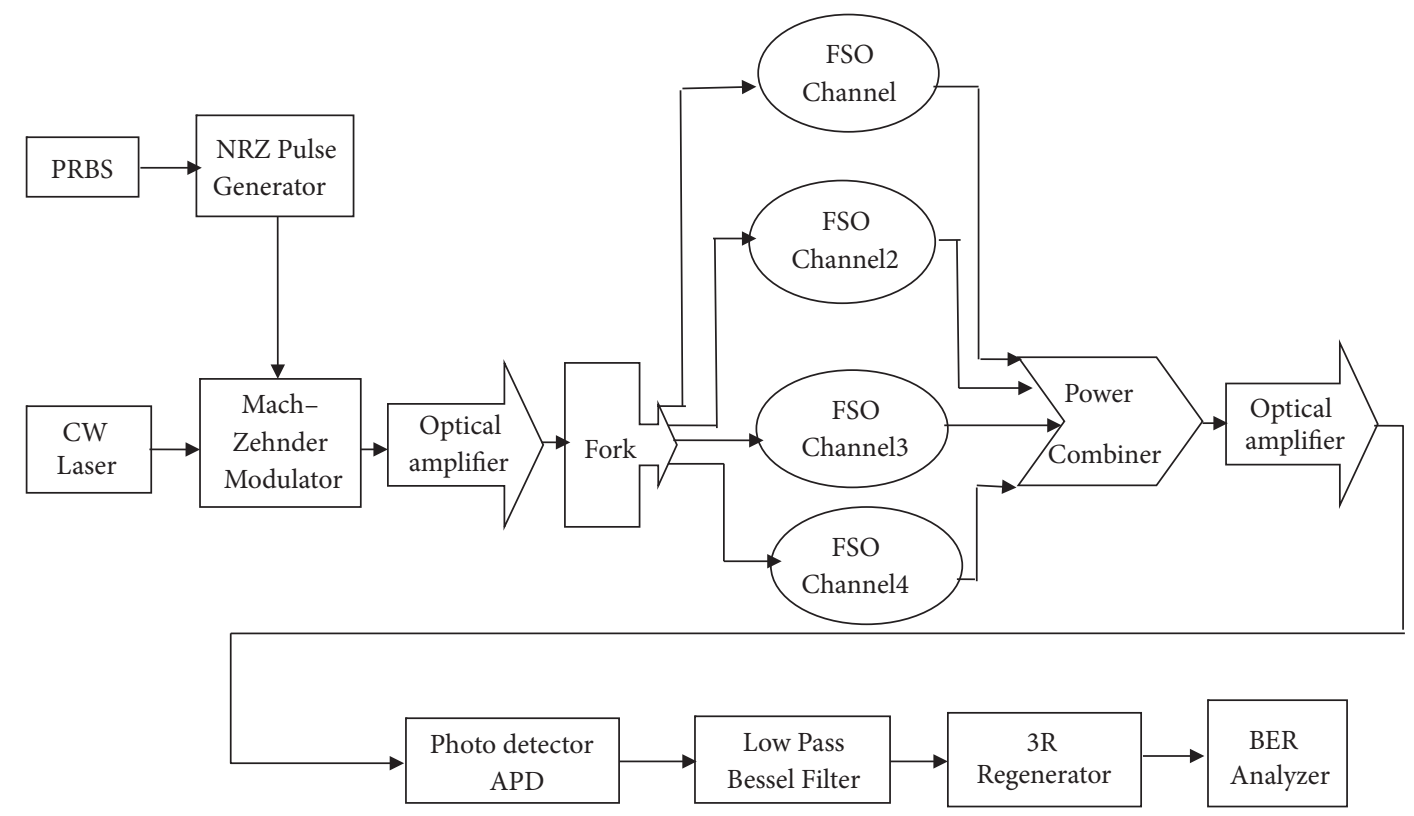

FIgURE 4: Simulation layout of $\left(4 \mathrm{~T}_{\mathrm{X}} / 4 \mathrm{R}_{\mathrm{X}}\right)$ FSO system.

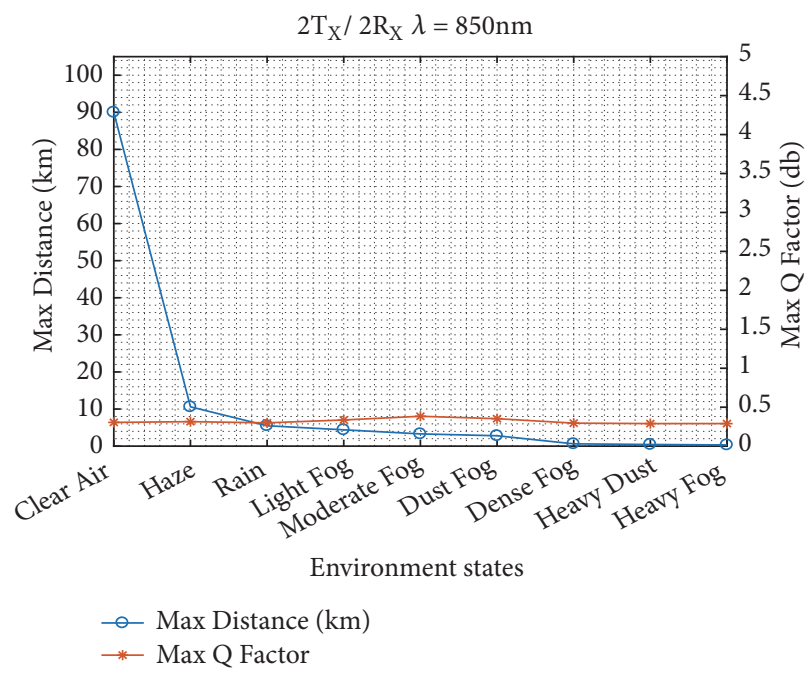

(a)

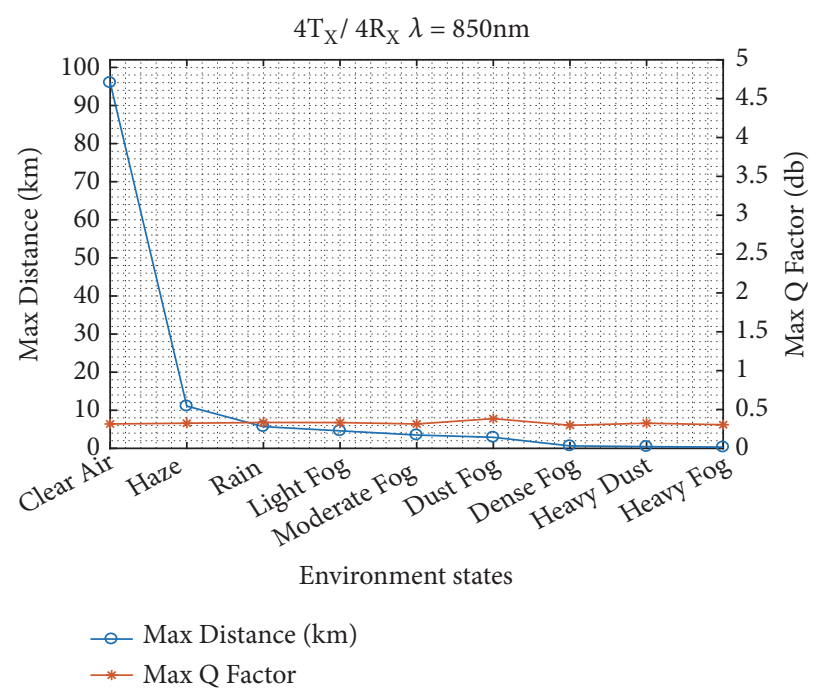

(b)

Figure 5: Relation between max distance $(\mathrm{km})$ and attenuation $(\mathrm{db} / \mathrm{km})$ at wavelength=850nm. (a) Using $2 \mathrm{~T}_{\mathrm{X}} / 2 \mathrm{R}_{\mathrm{X}}$; (b) using $4 \mathrm{~T}_{\mathrm{X}} / 4 \mathrm{R}_{\mathrm{X}}$.

It can reach a distance of $115 \mathrm{~km}$ for the $2 \mathrm{~T}_{\mathrm{X}} / 2 \mathrm{R}_{\mathrm{X}}$ with clear air, while at heavy fog the maximum range is about 0.375 $\mathrm{km}$.

In case of $4 \mathrm{~T}_{\mathrm{X}} / 4 \mathrm{R}_{\mathrm{X}}$, the maximum distance in the best case is $125 \mathrm{~km}$ while at worst case it is $390 \mathrm{~m}$.

In the best case one can reach a distance of $79 \mathrm{~km}$, while at worst case the maximum range achieved is $300 \mathrm{~m}$.

Using $2 \mathrm{~T}_{\mathrm{X}} / 2 \mathrm{R}_{\mathrm{X}}$ and $\lambda=850 \mathrm{~nm}$ at clear air the $\max$ distance is $90 \mathrm{~km}$ between transmitter and receiver while at heavy fog the max distance achieved between transmitter and receiver is $322 \mathrm{~m}$.

Using $4 \mathrm{~T}_{\mathrm{X}} / 4 \mathrm{R}_{\mathrm{X}}$ and $\lambda=850 \mathrm{~nm}$ the max distance achieved in the best conditions is $96 \mathrm{~km}$ while at worst case the max distance is $332 \mathrm{~m}$. The previous results in Tables 3-8 show the superiority of the $4 \mathrm{Tx} / 4 \mathrm{Rx}$ with wavelength of $1550 \mathrm{~nm}$ for Min BER, maximum Q factor, and max range between transmitter and receiver. Figures 5 and 6 show the relation between maximum range $(\mathrm{km})$ and attenuation $(\mathrm{db} / \mathrm{km})$ at different wavelengths $(850 \mathrm{~nm}-1550 \mathrm{~nm})$ using $2 \mathrm{~T}_{X} / 2 \mathrm{R}_{X}$ and $4 \mathrm{~T}_{\mathrm{X}} / 4 \mathrm{R}_{\mathrm{X}}$; for instance, the BER is no more than $10^{-9}$ and $\mathrm{Q}$ factor is at least 6 . We note that when attenuation increased (depend on weather conditions) max range decreased.

In Figure 5, it is obvious that, at wavelength $=850 \mathrm{~nm}$ using $2 \mathrm{~T}_{\mathrm{X}} / 2 \mathrm{R}_{\mathrm{X}}$ at clear air, the max distance obtained is $90 \mathrm{~km}$ between transmitter and receiver, while at heavy fog the max distance achieved between transmitter and receiver is $322 \mathrm{~m}$. 


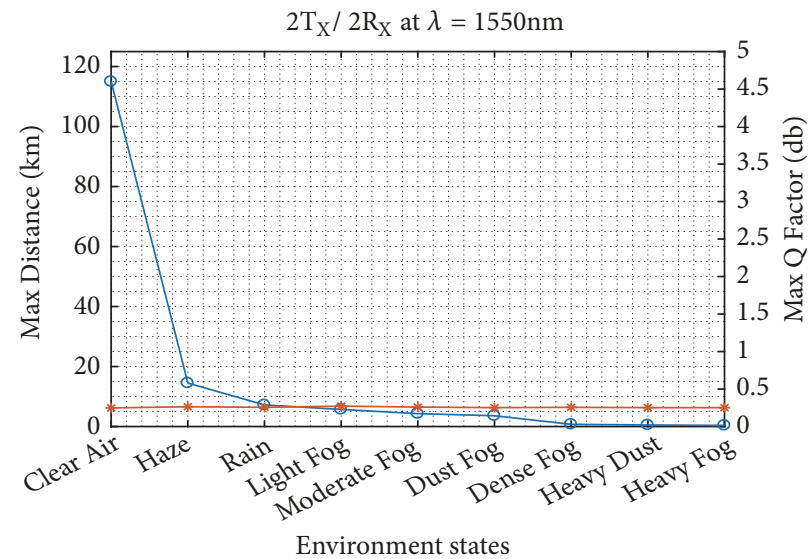

$\multimap$ Max Distance $(\mathrm{km})$

$\rightarrow$ Max Q Factor

(a)

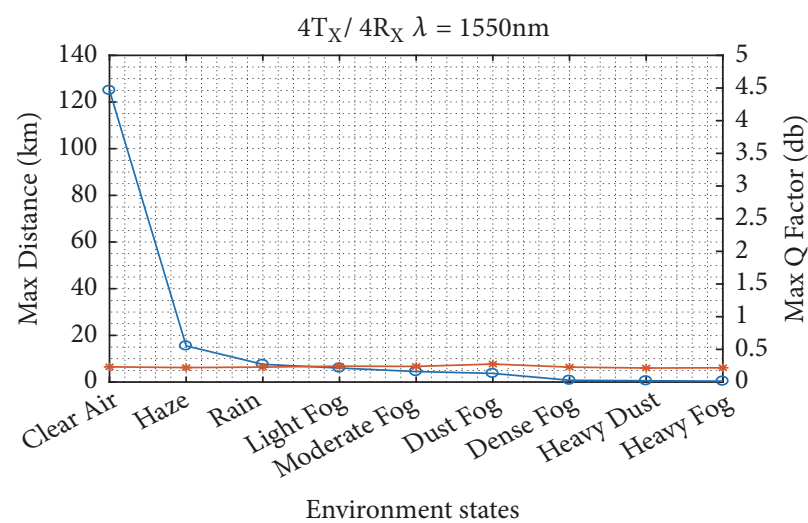

- Max Distance $(\mathrm{km})$

$\because$ Max Q Factor

FIGURE 6: Relation between max distance $(\mathrm{km})$ and attenuation $(\mathrm{db} / \mathrm{km})$ at wavelength=1550nm. (a) Using $2 \mathrm{~T}_{\mathrm{X}} / 2 \mathrm{R}_{\mathrm{X}}$; (b) $\mathrm{using} 4 \mathrm{~T}_{\mathrm{X}} / 4 \mathrm{R}_{\mathrm{X}}$.

TABLE 1: Global parameter setup.

\begin{tabular}{lc}
\hline Parameter & Value \\
\hline Transmission rate & $10 \mathrm{Gbps}$ \\
Transmission Wave length & $850 \mathrm{~nm}-1550 \mathrm{~nm}$ \\
Beam Divergence angle & $2 \mathrm{mrad}$ \\
Sequence length & $1024 \mathrm{bits}$ \\
Sample per bit & 64 \\
Number of sample & 65536 \\
Transmitter, Receiver aperture diameter & $10 \mathrm{~cm}, 45 \mathrm{~cm}$ \\
continuous wave laser power & $20 \mathrm{dbm}$ \\
Line width & $10 \mathrm{MHZ}$ \\
Transmitter, Receiver loss & $1 \mathrm{db}$ \\
Amplifier gain & $20 \mathrm{db}$ \\
APD responsivity & $1 \mathrm{~A} / \mathrm{W}$ \\
\hline
\end{tabular}

TABLE 2: Atmospheric attenuation at different weather conditions for $\lambda=(1550-850) \mathrm{nm}$ using Kruze model.

\begin{tabular}{lccc}
\hline $\begin{array}{l}\text { Weather } \\
\text { conditions }\end{array}$ & Visibility $(\mathrm{km})$ & $\begin{array}{c}\text { Attenuation }(\mathrm{db} / \mathrm{km}) \\
\text { At 1550nm }\end{array}$ & $\begin{array}{c}\text { Attenuation }(\mathrm{db} / \mathrm{km}) \\
\text { At 850nm }\end{array}$ \\
\hline Clear air & 23 & 0.2 & 0.42 \\
Haze & 2 & 2.8 & 5.3 \\
Rain & 1 & 6.5 & 11.3 \\
Light fog or & 0.8 & 8.5 & 14.4 \\
snow & & & 11.9 \\
Moderate fog or & 0.6 & 14.7 & 23.9 \\
heavy snow & 0.5 & 90 & 130 \\
Dust-fog & 0.1 & 132.8 & 188.2 \\
Dense fog & 0.07 & 190.9 & 266.5 \\
Heavy-dust & 0.05 & & 2 \\
Heavy-fog & & & \\
\hline
\end{tabular}


TABLE 3: Results for $1 \mathrm{~T}_{\mathrm{X}} / 1 \mathrm{R}_{\mathrm{X}}$ FSO at different weather conditions for $\lambda=1550 \mathrm{~nm}$.

\begin{tabular}{|c|c|c|c|}
\hline Weather conditions & Range $(\mathrm{km})$ & Max Q Factor & Min BER \\
\hline \multirow{3}{*}{$\begin{array}{l}\text { clear air } \\
\text { attenuation }=0.2 \mathrm{db} / \mathrm{km} \\
\text { visibility }=23 \mathrm{~km}\end{array}$} & 94 & 6.14 & $3.8 * 10^{\wedge}-10$ \\
\hline & 90 & 7.4 & $4.3 * 10^{\wedge}-14$ \\
\hline & 85 & 9.4 & $1.7 * 10^{\wedge}-21$ \\
\hline \multirow{3}{*}{$\begin{array}{l}\text { Haze } \\
\text { attenuation }=2.8 \mathrm{db} / \mathrm{km} \\
\text { visibility }=2 \mathrm{~km}\end{array}$} & 12.5 & 7.6 & $1.45 * 10^{\wedge}-14$ \\
\hline & 12 & 9.99 & $7.04 * 10^{\wedge}-24$ \\
\hline & 11.5 & 13.03 & $3.3 * 10^{\wedge}-39$ \\
\hline \multirow{3}{*}{$\begin{array}{l}\text { Rain } \\
\text { attenuation }=6.5 \mathrm{db} / \mathrm{km} \\
\text { visibility }=1 \mathrm{~km}\end{array}$} & 6.5 & 6.47 & $4.4 * 10^{\wedge}-11$ \\
\hline & 6 & 10.9 & $3.7 * 10^{\wedge}-28$ \\
\hline & 5.5 & 19.5 & $7.2 * 10^{\wedge}-85$ \\
\hline \multirow{3}{*}{$\begin{array}{l}\text { light fog } \\
\text { attenuation }=8.5 \mathrm{db} / \mathrm{km} \\
\text { visibility }=0.8 \mathrm{~km}\end{array}$} & 5.1 & 6.86 & $2.9 * 10^{\wedge}-12$ \\
\hline & 5 & 8.09 & $2.5 * 10^{\wedge}-16$ \\
\hline & 4.5 & 17.5 & $8.3 * 10^{\wedge}-69$ \\
\hline \multirow{3}{*}{$\begin{array}{l}\text { Moderate fog } \\
\text { attenuation }=11.9 \mathrm{db} / \mathrm{km} \\
\text { visibility }=0.6 \mathrm{~km}\end{array}$} & 3.9 & 6.06 & $6.08 * 10^{\wedge}-10$ \\
\hline & 3.5 & 14.6 & $6.5 * 10^{\wedge}-49$ \\
\hline & 3.4 & 17.9 & $1.6 * 10^{\wedge}-72$ \\
\hline \multirow{3}{*}{$\begin{array}{l}\text { dust fog } \\
\text { attenuation }=14.7 \mathrm{db} / \mathrm{km} \\
\text { visibility }=0.5 \mathrm{~km}\end{array}$} & 3.2 & 7.22 & $2.3 * 10^{\wedge}-13$ \\
\hline & 3 & 12.4 & $1.02 * 10^{\wedge}-35$ \\
\hline & 2.9 & 16.01 & $3.8 * 10^{\wedge}-58$ \\
\hline \multirow{3}{*}{$\begin{array}{l}\text { dense fog } \\
\text { attenuation }=90 \mathrm{db} / \mathrm{km} \\
\text { visibility }=0.1 \mathrm{~km}\end{array}$} & 0.675 & 6.5 & $4.6 * 10^{\wedge}-11$ \\
\hline & 0.65 & 9.7 & $1.1 * 10^{\wedge}-22$ \\
\hline & 0.6 & 20.5 & $6.06 * 10^{\wedge}-94$ \\
\hline \multirow{3}{*}{$\begin{array}{l}\text { heavy dust } \\
\text { attenuation }=132.8 \mathrm{db} / \mathrm{km} \\
\text { visibility }=0.07 \mathrm{~km}\end{array}$} & 0.48 & 6.17 & $2.9 * 10^{\wedge}-10$ \\
\hline & 0.45 & 12.5 & $3.5 * 10^{\wedge}-36$ \\
\hline & 0.43 & 19.2 & $9 * 10^{\wedge}-83$ \\
\hline \multirow{3}{*}{$\begin{array}{l}\text { heavy fog } \\
\text { attenuation }=190.9 \mathrm{db} / \mathrm{km} \\
\text { visibility }=0.05 \mathrm{~km}\end{array}$} & 0.345 & 6.6 & $1.4 * 10^{\wedge}-11$ \\
\hline & 0.34 & 7.9 & $1.3 * 10^{\wedge}-15$ \\
\hline & 0.335 & 9.3 & $4.4 * 10^{\wedge}-21$ \\
\hline
\end{tabular}

Using $4 \mathrm{~T}_{\mathrm{X}} / 4 \mathrm{R}_{\mathrm{X}}$ the max distance achieved in the best case is $96 \mathrm{~km}$ and at worst case the max distance is $332 \mathrm{~m}$.

In Figure 6, it is obvious that, at wavelength $=1550 \mathrm{~nm}$ using $2 \mathrm{~T}_{\mathrm{X}} / 2 \mathrm{R}_{\mathrm{X}}$ at clear air, the max distance obtained is 115 $\mathrm{km}$, while at heavy fog the max distance achieved between transmitter and receiver is $375 \mathrm{~m}$. Using $4 \mathrm{~T}_{\mathrm{X}} / 4 \mathrm{R}_{\mathrm{X}}$ the $\max$ distance achieved in the best case is $125 \mathrm{~km}$, while at worst case the max distance is $390 \mathrm{~m}$.

In Figure 7, it is obvious that when attenuation increases due to weather conditions, visibility decreases according to Table 2 . The best visibility at clear air will reach about $23 \mathrm{~km}$ while at heavy fog it is the worst visibility as it becomes $50 \mathrm{~m}$. It is clear that when we increase the wavelength of the transmitted signal the attenuation decreased. We take into consideration the case of heavy fog at different wavelengths $(850 \mathrm{~nm}-1550 \mathrm{~nm})$ using different channels and different distances (50, 100, 150, 200, 250, and $300 \mathrm{~m}$ ). Figures 8 and 9 show the relation between Max Q factor and different distances $(\mathrm{m})$ using different wavelengths and different channels.

In Figure 8, it is obvious that at distance $=300 \mathrm{~m}$ between transmitter and receiver and wavelength $=850 \mathrm{~nm}$ using $1 \mathrm{~T}_{\mathrm{X}} / 1 \mathrm{R}_{\mathrm{X}}$ the Max $\mathrm{Q}$ factor obtained=6.47, while using $2 \mathrm{~T}_{\mathrm{X}} / 2 \mathrm{R}_{\mathrm{X}}$ the Max $\mathrm{Q}$ factor obtained=16.23, and while using $4 \mathrm{~T}_{\mathrm{X}} / 4 \mathrm{R}_{\mathrm{X}}$ the Max $\mathrm{Q}$ factor obtained=24.5 (best result using $\left.4 \mathrm{~T}_{\mathrm{X}} / 4 \mathrm{R}_{\mathrm{X}}\right)$.

At distance of $300 \mathrm{~m}$ between transmitter and receiver with $\lambda=1550 \mathrm{~nm}$ using $1 \mathrm{~T}_{\mathrm{X}} / 1 \mathrm{R}_{\mathrm{X}}$ the Max $\mathrm{Q}$ factor obtained $=27.26$, while using $2 \mathrm{~T}_{\mathrm{X}} / 2 \mathrm{R}_{\mathrm{X}}$ the Max $\mathrm{Q}$ factor obtained $=58.76$, and while using $4 \mathrm{~T}_{X} / 4 \mathrm{R}_{X}$ the Max $\mathrm{Q}$ factor obtained $=84.8$ (best result using $4 \mathrm{~T}_{\mathrm{X}} / 4 \mathrm{R}_{\mathrm{X}}$ ) as shown in Figure 9. One can depict distances (from 0 to $150 \mathrm{~m}$ ); the value of $\mathrm{Q}$ factor at wavelength $=850$ is better than at wavelength $=1550 \mathrm{~nm}$, but at long distance the value of $\mathrm{Q}$ factor at wavelength $=1550$ is better than at wavelength $=850 \mathrm{~nm}$. So at long distance it is preferred to use wavelength $=1550 \mathrm{~nm}$. When we increase the power of laser above $20 \mathrm{dbm}$ at heavy fog we can improve the system performance as shown in Figures 10 and 11.

Figure 10 shows that increasing laser power to $25 \mathrm{dbm}$ at wavelength $=850 \mathrm{~nm}$ using $1 \mathrm{~T}_{\mathrm{X}} / 1 \mathrm{R}_{\mathrm{X}}$ the max distance obtained $=318 \mathrm{~m}$, while using $2 \mathrm{~T}_{\mathrm{X}} / 2 \mathrm{R}_{\mathrm{X}}$ the max distance obtained $=339 \mathrm{~m}$, and while using $4 \mathrm{~T}_{\mathrm{X}} / 4 \mathrm{R}_{\mathrm{X}}$ the max distance obtained $=350 \mathrm{~m}$ (best result using $4 \mathrm{~T}_{\mathrm{X}} / 4 \mathrm{R}_{\mathrm{X}}$ ). 
TABLE 4: Results for $2 \mathrm{~T}_{\mathrm{X}} / 2 \mathrm{R}_{\mathrm{X}}$ FSO at different weather conditions for $\lambda=1550 \mathrm{~nm}$.

\begin{tabular}{|c|c|c|c|}
\hline Weather conditions & Range $(\mathrm{km})$ & Max Q Factor & Min BER \\
\hline \multirow{3}{*}{$\begin{array}{l}\text { clear air } \\
\text { attenuation }=0.2 \mathrm{db} / \mathrm{km} \\
\text { visibility }=23 \mathrm{~km}\end{array}$} & 115 & 6.2 & $2.4 * 10^{\wedge}-10$ \\
\hline & 110 & 7.8 & $3.06 * 10^{\wedge}-15$ \\
\hline & 105 & 9.7 & $1.2 * 10^{\wedge}-22$ \\
\hline \multirow{3}{*}{$\begin{array}{l}\text { Haze } \\
\text { attenuation=2.8db } / \mathrm{km} \\
\text { visibility }=2 \mathrm{~km}\end{array}$} & 14.5 & 6.6 & $1.9 * 10^{\wedge}-11$ \\
\hline & 14 & 8.7 & $1.9 * 10^{\wedge}-18$ \\
\hline & 13.5 & 11.3 & $6.4 * 10^{\wedge}-30$ \\
\hline \multirow{3}{*}{$\begin{array}{l}\text { Rain } \\
\text { attenuation }=6.5 \mathrm{db} / \mathrm{km} \\
\text { visibility }=1 \mathrm{~km}\end{array}$} & 7.2 & 6.4 & $5.7 * 10^{\wedge}-11$ \\
\hline & 7 & 8.3 & $6.6 * 10^{\wedge}-17$ \\
\hline & 6.5 & 14.8 & $2.7 * 10^{\wedge}-50$ \\
\hline \multirow{3}{*}{$\begin{array}{l}\text { light fog } \\
\text { attenuation }=8.5 \mathrm{db} / \mathrm{km} \\
\text { visibility }=0.8 \mathrm{~km}\end{array}$} & 5.7 & 6.8 & $3.8 * 10^{\wedge}-12$ \\
\hline & 5.5 & 9.4 & $2.3 * 10^{\wedge}-21$ \\
\hline & 5 & 19.7 & $5.7 * 10^{\wedge}-87$ \\
\hline \multirow{3}{*}{$\begin{array}{l}\text { Moderate fog } \\
\text { attenuation }=11.9 \mathrm{db} / \mathrm{km} \\
\text { visibility }=0.6 \mathrm{~km}\end{array}$} & 4.3 & 6.5 & $3.5 * 10^{\wedge}-11$ \\
\hline & 4 & 12.5 & $2.7 * 10^{\wedge}-36$ \\
\hline & 3.8 & 18.7 & $7.6 * 10^{\wedge}-79$ \\
\hline \multirow{3}{*}{$\begin{array}{l}\text { dust fog } \\
\text { attenuation }=14.7 \mathrm{db} / \mathrm{km} \\
\text { visibility }=0.5 \mathrm{~km}\end{array}$} & 3.6 & 6.3 & $1.7 * 10^{\wedge}-10$ \\
\hline & 3.5 & 8.3 & $5.9 * 10^{\wedge}-17$ \\
\hline & 3.4 & 10.8 & $1.6 * 10^{\wedge}-27$ \\
\hline \multirow{3}{*}{$\begin{array}{l}\text { dense fog } \\
\text { attenuation }=90 \mathrm{db} / \mathrm{km} \\
\text { visibility }=0.1 \mathrm{~km}\end{array}$} & 0.735 & 6.4 & $7.6 * 10^{\wedge}-11$ \\
\hline & 0.7 & 11.18 & $2.11 * 10^{\wedge}-29$ \\
\hline & 0.65 & 23.1 & $1.02 * 10^{\wedge}-118$ \\
\hline \multirow{3}{*}{$\begin{array}{l}\text { heavy dust } \\
\text { attenuation }=132.8 \mathrm{db} / \mathrm{km} \\
\text { visibility }=0.07 \mathrm{~km}\end{array}$} & 0.52 & 6.3 & $1.8 * 10^{\wedge}-10$ \\
\hline & 0.5 & 10.04 & $4.2 * 10^{\wedge}-24$ \\
\hline & 0.48 & 15.6 & $2.6 * 10^{\wedge}-55$ \\
\hline \multirow{3}{*}{$\begin{array}{l}\text { heavy fog } \\
\text { attenuation }=190.9 \mathrm{db} / \mathrm{km} \\
\text { visibility }=0.05 \mathrm{~km}\end{array}$} & 0.375 & 6.3 & $1.6 * 10^{\wedge}-10$ \\
\hline & 0.37 & 7.45 & $3.8 * 10^{\wedge}-14$ \\
\hline & 0.35 & 14.3 & $1.6 * 10^{\wedge}-46$ \\
\hline
\end{tabular}

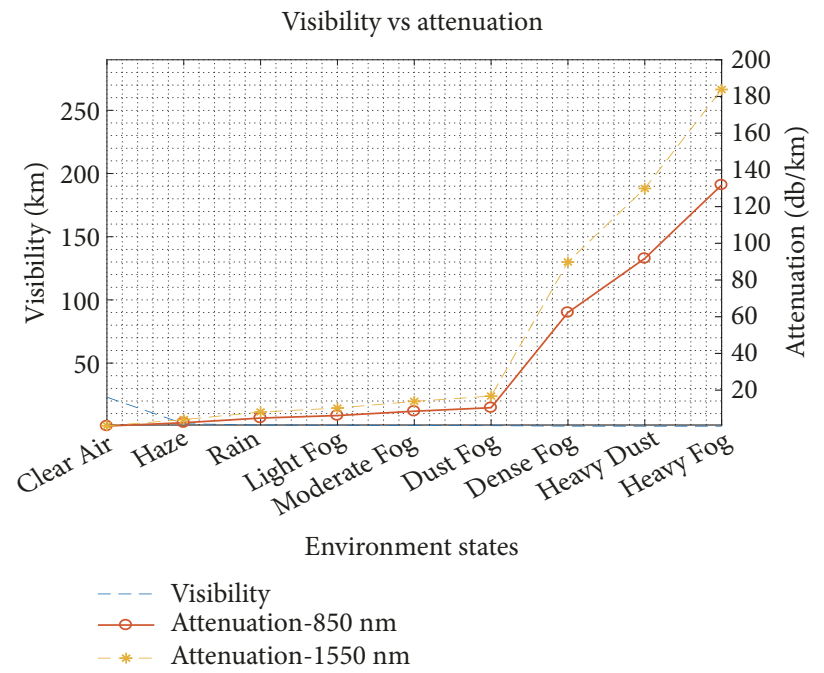

FIGURE 7: The attenuation $(\mathrm{db} / \mathrm{km})$ and visibility $(\mathrm{m})$ at different wavelengths $(850 \mathrm{~nm}-1550 \mathrm{~nm})$. 
TABLE 5: Results for $4 \mathrm{~T}_{X} / 4 \mathrm{R}_{X}$ FSO at different weather conditions for $\lambda=1550 \mathrm{~nm}$.

\begin{tabular}{|c|c|c|c|}
\hline Weather conditions & Range & Max Q Factor & Min BER \\
\hline \multirow{3}{*}{$\begin{array}{l}\text { clear air } \\
\text { attenuation }=0.2 \mathrm{db} / \mathrm{km} \\
\text { visibility }=23 \mathrm{~km}\end{array}$} & 125 & 6.5 & $3.3 * 10^{\wedge}-11$ \\
\hline & 0.12 & 8.1 & $2.2 * 10^{\wedge}-16$ \\
\hline & 0.11 & 12.4 & $1.4 * 10^{\wedge}-35$ \\
\hline \multirow{3}{*}{$\begin{array}{l}\text { Haze } \\
\text { attenuation }=2.8 \mathrm{db} / \mathrm{km} \\
\text { visibility }=2 \mathrm{~km}\end{array}$} & 15.5 & 6.2 & $2.5 * 10^{\wedge}-10$ \\
\hline & 15 & 8.15 & $1.5 * 10^{\wedge}-16$ \\
\hline & 14.5 & 10.6 & $1.11 * 10^{\wedge}-26$ \\
\hline \multirow{3}{*}{$\begin{array}{l}\text { Rain } \\
\text { attenuation }=6.5 \mathrm{db} / \mathrm{km} \\
\text { visibility }=1 \mathrm{~km}\end{array}$} & 7.6 & 6.4 & $8.4 * 10^{\wedge}-11$ \\
\hline & 7.5 & 7.2 & $2.3 * 10^{\wedge}-13$ \\
\hline & 7 & 13.06 & $2.05 * 10^{\wedge}-39$ \\
\hline \multirow{3}{*}{$\begin{array}{l}\text { light fog } \\
\text { attenuation }=8.5 \mathrm{db} / \mathrm{km} \\
\text { visibility }=0.8 \mathrm{~km}\end{array}$} & 6 & 6.8 & $3.3 * 10^{\wedge}-12$ \\
\hline & 5.8 & 9.4 & $2.2 * 10^{\wedge}-21$ \\
\hline & 5.6 & 12.7 & $1.6 * 10^{\wedge}-37$ \\
\hline \multirow{3}{*}{$\begin{array}{l}\text { Moderate fog } \\
\text { attenuation }=11.9 \mathrm{db} / \mathrm{km} \\
\text { visibility }=0.6 \mathrm{~km}\end{array}$} & 4.5 & 6.7 & $6.01 * 10^{\wedge}-12$ \\
\hline & 4.3 & 10.5 & $4.6 * 10^{\wedge}-26$ \\
\hline & 4 & 19.25 & $5.2 * 10^{\wedge}-83$ \\
\hline \multirow{3}{*}{$\begin{array}{l}\text { dust fog } \\
\text { attenuation }=14.7 \mathrm{db} / \mathrm{km} \\
\text { visibility }=0.5 \mathrm{~km}\end{array}$} & 3.7 & 7.7 & $4.05 * 10^{\wedge}-15$ \\
\hline & 3.5 & 13.08 & $1.6 * 10^{\wedge}-39$ \\
\hline & 3.4 & 16.7 & $1.7 * 10^{\wedge}-63$ \\
\hline \multirow{3}{*}{$\begin{array}{l}\text { dense fog } \\
\text { attenuation }=90 \mathrm{db} / \mathrm{km} \\
\text { visibility }=0.1 \mathrm{~km}\end{array}$} & 0.765 & 6.4 & $8.4 * 10^{\wedge}-11$ \\
\hline & 0.75 & 8.14 & $1.7 * 10^{\wedge}-16$ \\
\hline & 0.74 & 9.5 & $6.2 * 10^{\wedge}-22$ \\
\hline \multirow{3}{*}{$\begin{array}{l}\text { heavy dust } \\
\text { attenuation }=132.8 \mathrm{db} / \mathrm{km} \\
\text { visibility=0.07 km }\end{array}$} & 0.542 & 6 & $8.4 * 10^{\wedge}-10$ \\
\hline & 0.52 & 10.1 & $2.1 * 10^{\wedge}-24$ \\
\hline & 0.5 & 15.7 & $8.4 * 10^{\wedge}-56$ \\
\hline \multirow{3}{*}{$\begin{array}{l}\text { heavy fog } \\
\text { attenuation }=190.9 \mathrm{db} / \mathrm{km} \\
\text { visibility }=0.05 \mathrm{~km}\end{array}$} & 0.39 & 6.11 & $4.35 * 10^{\wedge}-10$ \\
\hline & 0.38 & 8.6 & $3.3 * 10^{\wedge}-18$ \\
\hline & 0.37 & 11.9 & $5.11 * 10^{\wedge}-33$ \\
\hline
\end{tabular}

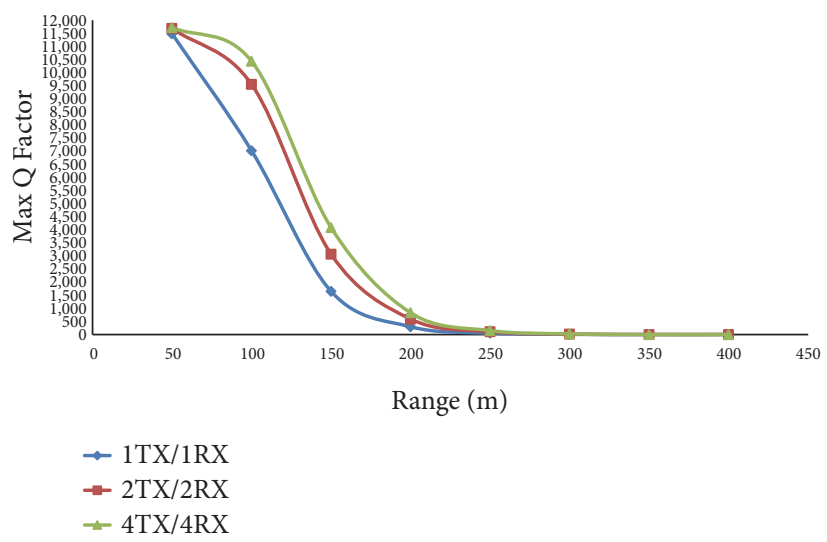

Figure 8: Max Q factor and range (m) at $\lambda$ of $850 \mathrm{~nm}$ and attenuation of $266.5 \mathrm{db} / \mathrm{km}$ (heavy fog).

Increasing laser power to $25 \mathrm{dbm}$ at wavelength $=1550$ $\mathrm{nm}$ using $1 \mathrm{~T}_{\mathrm{X}} / 1 \mathrm{R}_{\mathrm{X}}$, the max distance obtained $=370 \mathrm{~m}$, while using $2 \mathrm{~T}_{\mathrm{X}} / 2 \mathrm{R}_{\mathrm{X}}$ the max distance obtained $=400 \mathrm{~m}$, and while using $4 \mathrm{~T}_{\mathrm{X}} / 4 \mathrm{R}_{\mathrm{X}}$ the max distance obtained $=414 \mathrm{~m}$ as shown in Figure 11.

\section{Conclusion}

The influence of environmental parameters such as rain, fog, haze, snow, and dust on free space optical links has been simulated and discussed. According to obtained results, the 
TABLE 6: Results for $1 \mathrm{~T}_{\mathrm{X}} / 1 \mathrm{R}_{\mathrm{X}} \mathrm{FSO}$ at different weather conditions for $850 \mathrm{~nm}$ wavelength.

\begin{tabular}{|c|c|c|c|}
\hline Weather conditions & Range (km) & Max Q Factor & Min BER \\
\hline \multirow{3}{*}{$\begin{array}{l}\text { clear air } \\
\text { attenuation }=0.42 \mathrm{db} / \mathrm{km} \\
\text { visibility }=23 \mathrm{~km}\end{array}$} & 79 & 6.2 & $3.4 * 10^{\wedge}-10$ \\
\hline & 75 & 8.7 & $1.5 * 10^{\wedge}-18$ \\
\hline & 70 & 13.08 & $1.6 * 10^{\wedge}-39$ \\
\hline \multirow{3}{*}{$\begin{array}{l}\text { haze } \\
\text { attenuation }=5.3 \mathrm{db} / \mathrm{km} \\
\text { visibility }=2 \mathrm{~km}\end{array}$} & 9.7 & 6.1 & $4.7 * 10^{\wedge}-10$ \\
\hline & 9 & 12.1 & $3.7 * 10^{\wedge}-34$ \\
\hline & 8.5 & 18.9 & $8.5 * 10^{\wedge}-81$ \\
\hline \multirow{3}{*}{$\begin{array}{l}\text { rain } \\
\text { attenuation=11.3 db/km } \\
\text { visibility }=1 \mathrm{~km}\end{array}$} & 5 & 6.7 & $5.8 * 10^{\wedge}-12$ \\
\hline & 4.7 & 12.4 & $5.3 * 10^{\wedge}-36$ \\
\hline & 4.5 & 18.2 & $4.1 * 10^{\wedge}-74$ \\
\hline \multirow{3}{*}{$\begin{array}{l}\text { light fog } \\
\text { attenuation }=14.4 \mathrm{db} / \mathrm{km} \\
\text { visibility }=0.8 \mathrm{~km}\end{array}$} & 4 & 7.7 & $4.8 * 10^{\wedge}-15$ \\
\hline & 3.9 & 10.02 & $5.2 * 10^{\wedge}-24$ \\
\hline & 3.8 & 12.8 & $3.2 * 10^{\wedge}-38$ \\
\hline \multirow{3}{*}{$\begin{array}{l}\text { Moderate fog } \\
\text { attenuation }=19.7 \mathrm{db} / \mathrm{km} \\
\text { visibility }=0.6 \mathrm{~km}\end{array}$} & 3.1 & 6.3 & $1.7 * 10^{\wedge}-10$ \\
\hline & 3 & 9 & $9.7 * 10^{\wedge}-20$ \\
\hline & 2.9 & 12.7 & $3.4 * 10^{\wedge}-37$ \\
\hline \multirow{3}{*}{$\begin{array}{l}\text { dust fog } \\
\text { attenuation }=23.9 \mathrm{db} / \mathrm{km} \\
\text { visibility }=0.5 \mathrm{~km}\end{array}$} & 2.6 & 6.7 & $7.9 * 10^{\wedge}-12$ \\
\hline & 2.5 & 10.3 & $1.8 * 10^{\wedge}-25$ \\
\hline & 2.4 & 15.5 & $1.8 * 10^{\wedge}-54$ \\
\hline \multirow{3}{*}{$\begin{array}{l}\text { dense fog } \\
\text { attenuation }=130 \mathrm{db} / \mathrm{km} \\
\text { visibility }=0.1 \mathrm{~km}\end{array}$} & 0.579 & 6.05 & $6.6 * 10^{\wedge}-10$ \\
\hline & 0.55 & 11.7 & $4.91 * 10^{\wedge}-32$ \\
\hline & 0.54 & 14.5 & $7.8 * 10^{\wedge}-48$ \\
\hline \multirow{3}{*}{$\begin{array}{l}\text { heavy dust } \\
\text { attenuation }=188.2 \mathrm{db} / \mathrm{km} \\
\text { visibility }=0.07 \mathrm{~km}\end{array}$} & 0.41 & 6.9 & $1.8 * 10^{\wedge}-12$ \\
\hline & 0.4 & 9.6 & $2.3 * 10^{\wedge}-22$ \\
\hline & 0.39 & 13.2 & $5.6 * 10^{\wedge}-40$ \\
\hline \multirow{3}{*}{$\begin{array}{l}\text { heavy fog } \\
\text { attenuation }=266.5 \mathrm{db} / \mathrm{km} \\
\text { visibility }=0.05 \mathrm{~km}\end{array}$} & 0.3 & 6.5 & $4.2 * 10^{\wedge}-11$ \\
\hline & 0.29 & 10.29 & $3.1 * 10^{\wedge}-25$ \\
\hline & 0.28 & 15.8 & $8.4 * 10^{\wedge}-57$ \\
\hline
\end{tabular}

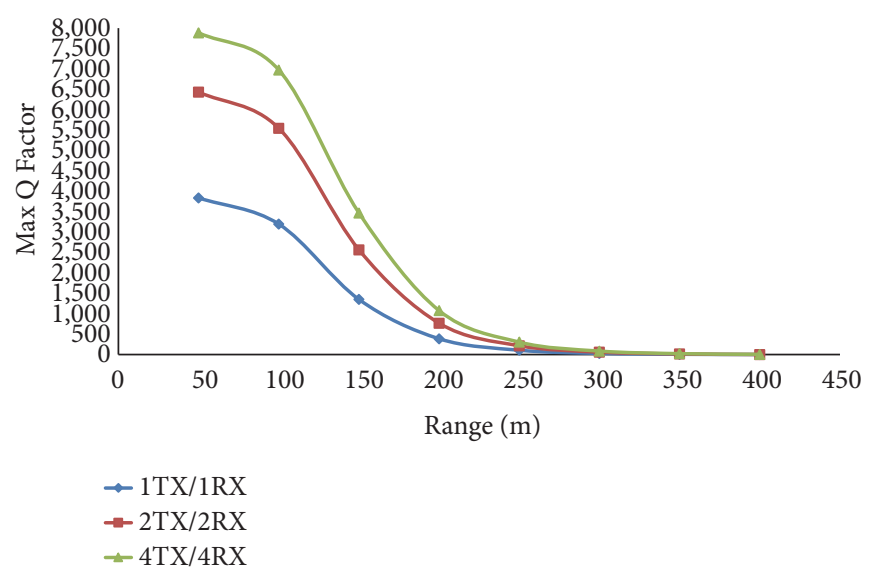

Figure 9: Max Q factor and range (m) at $\lambda=1550 \mathrm{~nm}$ and attenuation=190.9 db/km (heavy fog).

MIMO techniques have been used to enhance the system performance. In this case optimal design has been done by using four transmitters and four receivers $(4 \mathrm{Tx} / 4 \mathrm{Rx})$ free space optical system with wavelength of $1550 \mathrm{~nm}$. The obtained results have been confirming the significantly dependence between the atmospheric attenuation, weather condition, and operational wavelengths. The system performance at wave length of $1550 \mathrm{~nm}$ is much better than $850 \mathrm{~nm}$ wave length. In this paper, results for the Min BER, Max Q factor, and max range have been numerically evaluated and presented with 
TABLE 7: Results for $2 \mathrm{~T}_{\mathrm{X}} / 2 \mathrm{R}_{\mathrm{X}}$ FSO at different weather conditions for $850 \mathrm{~nm}$ wavelength.

\begin{tabular}{|c|c|c|c|}
\hline Weather conditions & Range & Max Q Factor & Min BER \\
\hline \multirow{3}{*}{$\begin{array}{l}\text { clear air } \\
\text { attenuation }=0.42 \mathrm{db} / \mathrm{km} \\
\text { visibility }=23 \mathrm{~km}\end{array}$} & 90 & 6.4 & $5.5 * 10^{\wedge}-11$ \\
\hline & 85 & 9.7 & $8.2 * 10^{\wedge}-23$ \\
\hline & 80 & 14.4 & $1.9 * 10^{\wedge}-47$ \\
\hline \multirow{3}{*}{$\begin{array}{l}\text { Haze } \\
\text { attenuation }=5.3 \mathrm{db} / \mathrm{km} \\
\text { visibility }=2 \mathrm{~km}\end{array}$} & 10.6 & 6.6 & $1.7 * 10^{\wedge}-11$ \\
\hline & 10.5 & 7.3 & $1.2 * 10^{\wedge}-13$ \\
\hline & 10 & 11.77 & $2.13 * 10^{\wedge}-32$ \\
\hline \multirow{3}{*}{$\begin{array}{l}\text { Rain } \\
\text { attenuation }=11.3 \mathrm{db} / \mathrm{km} \\
\text { visibility }=1 \mathrm{~km}\end{array}$} & 5.5 & 6.28 & $1.5 * 10^{\wedge}-10$ \\
\hline & 5 & 16.8 & $4.05 * 10^{\wedge}-64$ \\
\hline & 4.9 & 20.2 & $4.3 * 10^{\wedge}-91$ \\
\hline \multirow{3}{*}{$\begin{array}{l}\text { light fog } \\
\text { attenuation }=14.4 \mathrm{db} / \mathrm{km} \\
\text { visibility }=0.8 \mathrm{~km}\end{array}$} & 4.4 & 7.06 & $7.4 * 10^{\wedge}-13$ \\
\hline & 4.3 & 9.2 & $2.1 * 10^{\wedge}-20$ \\
\hline & 4 & 18.88 & $5.98 * 10^{\wedge}-80$ \\
\hline \multirow{3}{*}{$\begin{array}{l}\text { Moderate fog } \\
\text { attenuation }=19.7 \mathrm{db} / \mathrm{km} \\
\text { visibility }=0.6 \mathrm{~km}\end{array}$} & 3.3 & 8.05 & $3.5 * 10^{\wedge}-16$ \\
\hline & 3.2 & 11.4 & $2.4 * 10^{\wedge}-30$ \\
\hline & 3 & 21.5 & $2.08 * 10^{\wedge}-103$ \\
\hline \multirow{3}{*}{$\begin{array}{l}\text { dust fog } \\
\text { attenuation }=23.9 \mathrm{db} / \mathrm{km} \\
\text { visibility }=0.5 \mathrm{~km}\end{array}$} & 2.8 & 7.4 & $4.9 * 10^{\wedge}-14$ \\
\hline & 2.6 & 16.7 & $1.9 * 10^{\wedge}-63$ \\
\hline & 2.5 & 24.3 & $2.5 * 10^{\wedge}-131$ \\
\hline \multirow{3}{*}{$\begin{array}{l}\text { dense fog } \\
\text { attenuation }=130 \mathrm{db} / \mathrm{km} \\
\text { visibility }=0.1 \mathrm{~km}\end{array}$} & 0.62 & 6.2 & $2.7 * 10^{\wedge}-10$ \\
\hline & 0.6 & 9.8 & $4.8 * 10^{\wedge}-23$ \\
\hline & 0.58 & 15 & $3 * 10^{\wedge}-51$ \\
\hline \multirow{3}{*}{$\begin{array}{l}\text { heavy dust } \\
\text { attenuation }=188.2 \mathrm{db} / \mathrm{km} \\
\text { visibility }=0.07 \mathrm{~km}\end{array}$} & 0.443 & 6.08 & $5.35 * 10^{\wedge}-10$ \\
\hline & 043 & 9.4 & $3.2 * 10^{\wedge}-21$ \\
\hline & 0.425 & 10.97 & $2.3 * 10^{\wedge}-28$ \\
\hline \multirow{3}{*}{$\begin{array}{l}\text { heavy fog } \\
\text { attenuation }=266.5 \mathrm{db} / \mathrm{km} \\
\text { visibility }=0.05 \mathrm{~km}\end{array}$} & 0.322 & 6.08 & $5.2 * 10^{\wedge}-10$ \\
\hline & 0.31 & 10.6 & $1.2 * 10^{\wedge}-26$ \\
\hline & 0.3 & 16.2 & $1.2 * 10^{\wedge}-59$ \\
\hline
\end{tabular}

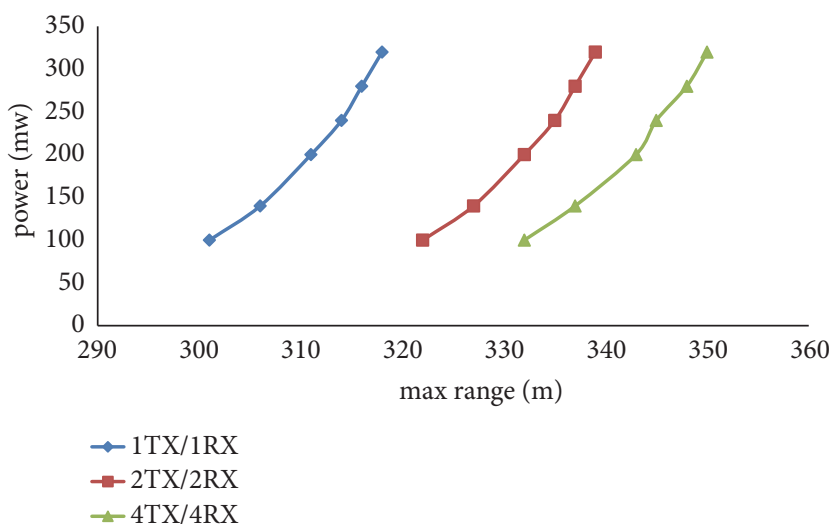

Figure 10: Power (mw) and max range $(\mathrm{m})$ at wavelength $=850 \mathrm{~nm}$ and attenuation $=266.5 \mathrm{db} / \mathrm{km}$ (heavy fog). 
TABLE 8: Results for $4 \mathrm{~T}_{\mathrm{X}} / 4 \mathrm{R}_{\mathrm{X}} \mathrm{FSO}$ at different weather conditions for $\lambda=850 \mathrm{~nm}$.

\begin{tabular}{|c|c|c|c|}
\hline Weather conditions & Range $(\mathrm{km})$ & Max Q Factor & Min BER \\
\hline \multirow{3}{*}{$\begin{array}{l}\text { Clear Air } \\
\text { Attenuation }=0.42 \mathrm{db} / \mathrm{Km} \\
\text { Visibility }=23 \mathrm{~km}\end{array}$} & 96 & 6.4 & $8.9 * 10^{\wedge}-11$ \\
\hline & 95 & 6.9 & $1.9 * 10^{\wedge}-12$ \\
\hline & 90 & 10.4 & $1.2 * 10^{\wedge}-25$ \\
\hline \multirow{3}{*}{$\begin{array}{l}\text { Haze } \\
\text { Attenuation }=5.3 \mathrm{db} / \mathrm{Km} \\
\text { Visibility=2 Km }\end{array}$} & 11.1 & 6.6 & $2.2 * 10^{\wedge}-11$ \\
\hline & 10.5 & 11.7 & $7.6 * 10^{\wedge}-32$ \\
\hline & 10 & 18.15 & $4.6 * 10^{\wedge}-74$ \\
\hline \multirow{3}{*}{$\begin{array}{l}\text { Rain } \\
\text { Attenuation }=11.3 \mathrm{db} / \mathrm{Km} \\
\text { Visibility }=1 \mathrm{Km}\end{array}$} & 5.7 & 6.8 & $6.2 * 10^{\wedge}-12$ \\
\hline & 5.6 & 8.3 & $4 * 10^{\wedge}-17$ \\
\hline & 5.5 & 10.15 & $1.3 * 10^{\wedge}-24$ \\
\hline \multirow{3}{*}{$\begin{array}{l}\text { Light Fog } \\
\text { Attenuation }=14.4 \mathrm{db} / \mathrm{Km} \\
\text { Visibility }=0.8 \mathrm{Km}\end{array}$} & 4.6 & 6.77 & $5.5 * 10^{\wedge}-12$ \\
\hline & 4.5 & 8.8 & 5. $8 * 10^{\wedge}-19$ \\
\hline & 4.4 & 11.3 & $5.2 * 10^{\wedge}-30$ \\
\hline \multirow{3}{*}{$\begin{array}{l}\text { Moderate Fog } \\
\text { Attenuation }=19.7 \mathrm{db} / \mathrm{Km} \\
\text { Visibility }=0.6 \mathrm{Km}\end{array}$} & 3.5 & 6.4 & $8 * 10^{\wedge}-11$ \\
\hline & 3.4 & 9.11 & $3.4 * 10^{\wedge}-20$ \\
\hline & 3.3 & 12.7 & $1.1 * 10^{\wedge}-37$ \\
\hline \multirow{3}{*}{$\begin{array}{l}\text { Dust Fog } \\
\text { Attenuation }=23.9 \mathrm{db} / \mathrm{Km} \\
\text { Visibility }=0.5 \mathrm{Km}\end{array}$} & 2.9 & 7.8 & $2.3 * 10^{\wedge}-15$ \\
\hline & 2.8 & 11.8 & $9.9 * 10^{\wedge}-33$ \\
\hline & 2.7 & 17.5 & $8.6 * 10^{\wedge}-69$ \\
\hline \multirow{3}{*}{$\begin{array}{l}\text { Dense Fog } \\
\text { Attenuation }=130 \mathrm{db} / \mathrm{Km} \\
\text { Visibility }=0.1 \mathrm{Km}\end{array}$} & 0.642 & 6.06 & $6.16 * 10^{\wedge}-10$ \\
\hline & 0.63 & 8 & $4.7 * 10^{\wedge}-16$ \\
\hline & 0.62 & 10.02 & $5.14 * 10^{\wedge}-24$ \\
\hline \multirow{3}{*}{$\begin{array}{l}\text { Heavy Dust } \\
\text { Attenuation }=188.2 \mathrm{db} / \mathrm{Km} \\
\text { Visibility }=0.07 \mathrm{Km}\end{array}$} & 0.455 & 6.6 & $1.3 * 10^{\wedge}-11$ \\
\hline & 0.45 & 7.9 & $1.6 * 10^{\wedge}-15$ \\
\hline & 0.44 & 10.8 & $1.04 * 10^{\wedge}-27$ \\
\hline \multirow{3}{*}{$\begin{array}{l}\text { Heavy Fog } \\
\text { Attenuation }=266.5 \mathrm{db} / \mathrm{Km} \\
\text { Visibility }=0.05 \mathrm{Km}\end{array}$} & 0.332 & 6.2 & $2.4 * 10^{\wedge}-10$ \\
\hline & 0.32 & 10.77 & $1.8 * 10^{\wedge}-27$ \\
\hline & 0.315 & 13.4 & $3.7 * 10^{\wedge}-41$ \\
\hline
\end{tabular}

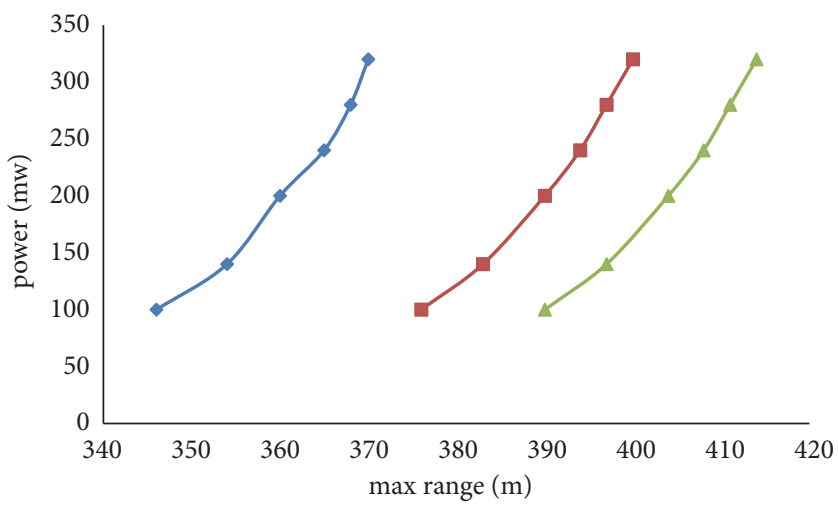

$$
\begin{aligned}
& \rightarrow 1 \mathrm{TX} / 1 \mathrm{RX} \\
& \rightarrow 2 \mathrm{TX} / 2 \mathrm{RX} \\
& \leftarrow 4 \mathrm{TX} / 4 \mathrm{RX}
\end{aligned}
$$

Figure 11: Power (mw) and max range (m) at $\lambda=1550 \mathrm{~nm}$ and $\alpha=190.9 \mathrm{db} / \mathrm{km}$ (heavy fog). 
different wavelengths (1550nm-850nm). The system BER is lower than $10^{-9}$ and $\mathrm{Q}$ factor is at least 6 . The max range allowed to achieve in case of heavy fog is $390 \mathrm{~m}$ and $332 \mathrm{~m}$ at wave lengths of $1550 \mathrm{~nm}$ and $850 \mathrm{~nm}$, respectively.

\section{Data Availability}

The data used to support the findings of this study are available from the corresponding author upon request.

\section{Conflicts of Interest}

The authors declare that they have no conflicts of interest.

\section{References}

[1] K. Singh and M. S. Bhamrah, "Investigations on aperture diameter of telescope in intersatellite optical wireless communication system," International Journal of Engineering Research and Applications (IJERA), vol. 2, no. 3, pp. 1660-1670, 2012.

[2] A. Gupta, "Comparative analysis of free space optical communication system for various optical transmission windows under adverse weather conditions," ELSEVIER Procedia Computer Science, vol. 89, pp. 99-106, 2016.

[3] E. M. Reddy and A. B. Therese, "Analysis of atmospheric effects on free space optical communication," in Proceedings of the International Conference on Next gen Electronic Technologies: Silicon to Software (ICNETS2), pp. 338-343, 2017.

[4] Z. N. Chaleshtory, A. Gholami, Z. Ghassemlooy, and M. Sedghi, "Experimental investigation of environment effects on the FSO link with turbulence," IEEE Photonics Technology Letters, vol. 29, no. 17, pp. 1435-1438, 2017.

[5] A. Touati, A. Abdaoui, F. Touati, M. Uysal, and A. Bouallegue, "On the effects of temperature on the performances of fso transmission under qatar's climate," in Proceedings of the 85th IEEE Vehicular Technology Conference, VTC Spring 2017, June 2017.

[6] M. F. Abdul-Zahra, M. I. Abdullah, and A. RajiJabbar, "Dust effect on the performance of optical wireless communication system," Journal of University of Babylon, vol. 26, no. 1, pp. 259268, 2018.

[7] N. B. M. D. Ishak and A. B. B. Ibrahim, "the effect of atmosphere conditions on performance of free space optics in malaysia at $1550 \mathrm{~nm}$," International Journal of Advances in Science Engineering and Technology, vol. 3, no. 3, pp. 29-34, 2015.

[8] A. SreeMadhuri and S. T. Mahaboob, "Evaluating the performance of free space optical link in tropical climate," International Journal of Advance Engineering and ResearchDevelopment, vol. 4, no. 8, pp. 273-278, 2017.

[9] M. N. Islam and M. N. Al Safa Bhuiyan, "Effect of operating wavelengths and different weather conditions on performance of point-to-point free space optical link," International Journal of Computer Networks \& Communications, vol. 8, no. 2, pp. 6375, 2016.

[10] S. A. KadhimP, S. A. A. Taha, and A. Q. Baki, "Characterization study and simulation of MIMO free space optical communication under different atmospheric channel," International Journal of Innovative Science, Engineering \& Technology, vol. 3, no. 8, pp. 587-595., 2016.
[11] G. Soni, "Performance Analysis of Free Space Optical Link Under Various Attenuation Effects," Science Journal of Circuits, Systems and Signal Processing, vol. 7, no. 2, p. 43, 2018.

[12] N. Hameed, T. M. Jatoi, and H. U. Manzoor, "Effect of Weather Conditions on FSO link based in Islamabad," in Proceedings of the 2014 IEEE 17th International Multi-Topic Conference (INMIC), pp. 1-5, Research Gate Conference Paper, 2014.

[13] S. H. Alnajjar, A. A. Noori, and A. A. Moosa, "Enhancement of FSO communications links under complex environment," Photonic Sensors, vol. 7, no. 2, pp. 113-122, 2017.

[14] M. Uysal, C. Capsoni, Z. Ghassemlooy, A. Boucouvalas, and E. Udvary, Eds., Optical Wireless Communications: An Emerging Technology, Springer, 2016.

[15] S. A. Kadhim, A. A. J. Shakir, A. N. Mohammad, and N. F. Mohammad, "System design and simulation using(optisystem 7.0) for performance characterization of the free space optical communication system," International Journal of Innovative Research in Science, Engineering and Technology, vol. 4, no. 6, pp. 4823-4831, 2015.

[16] H. Kaur and H. Sarangal, "Simulative investigation of FSO system using $4 \mathrm{X} 4$ transmitter receiver combination integrated with various types of amplifiers under different weather conditions," International Journal of Signal Processing, Image Processing and Pattern Recognition, vol. 9, no. 3, pp. 11-16, 2016.

[17] A. Malik and P. Singh, "Free space optics: current applications and future challenges," International Journal of Optics, vol. 2015, Article ID 945483, 7 pages, 2015.

[18] J. W. Lai, Free space optical communication for tactical operations, vol. 2, Institutional archive of the naval postgraduate school, 2016. 

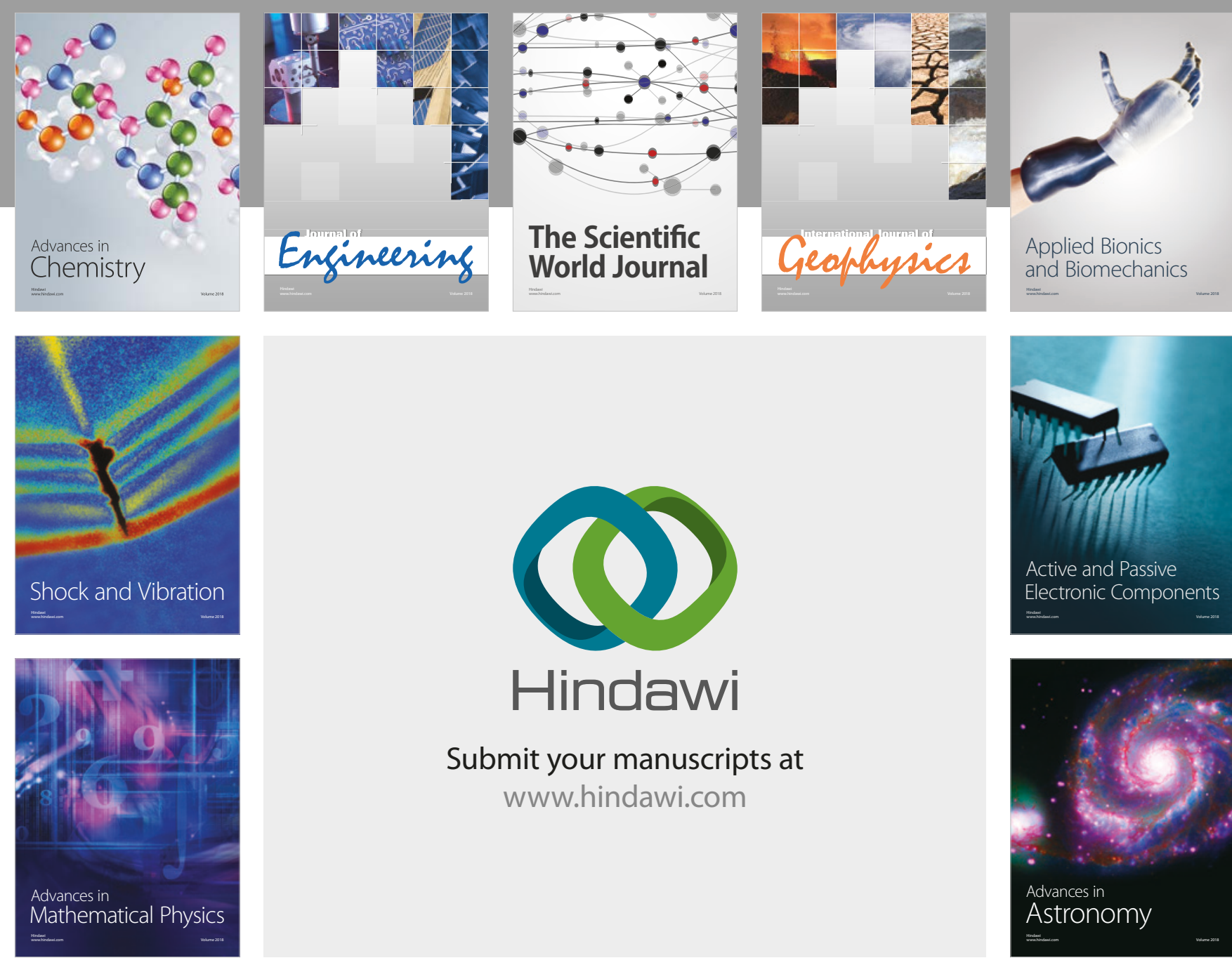

Submit your manuscripts at

www.hindawi.com

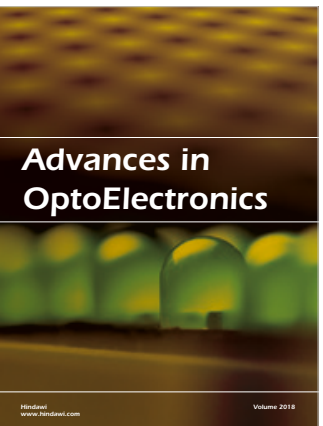

\section{Rotcting Machinery}
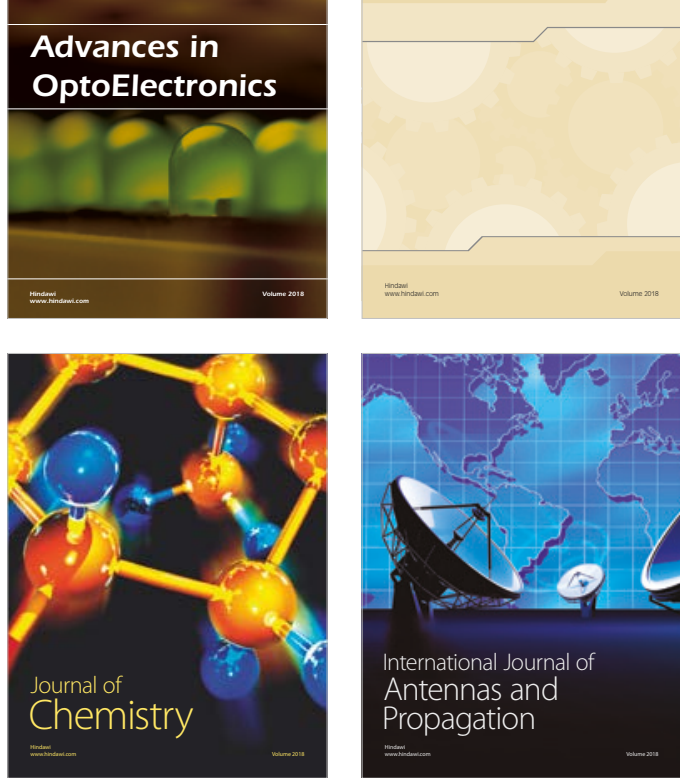

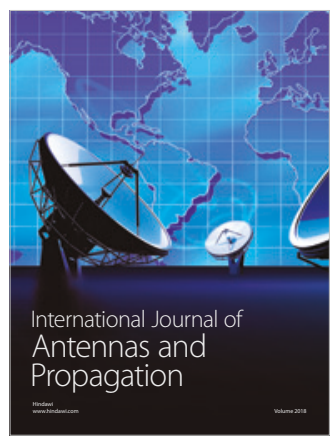

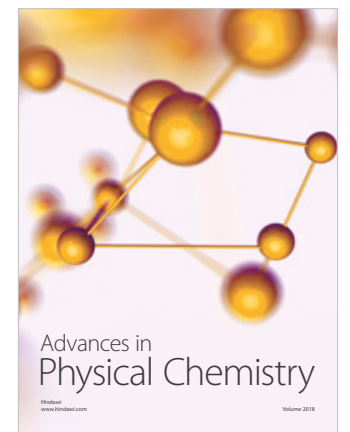

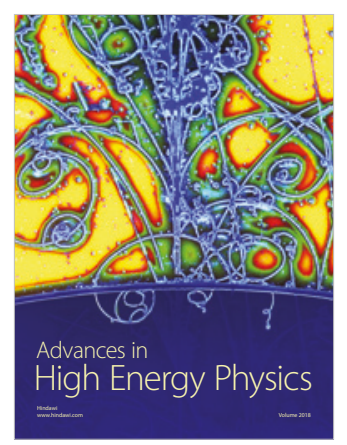

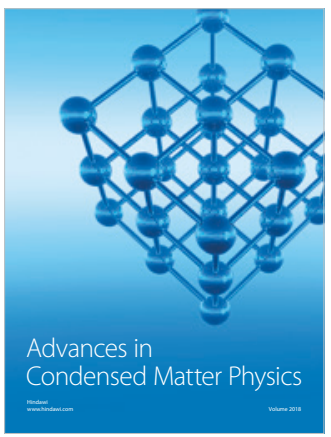

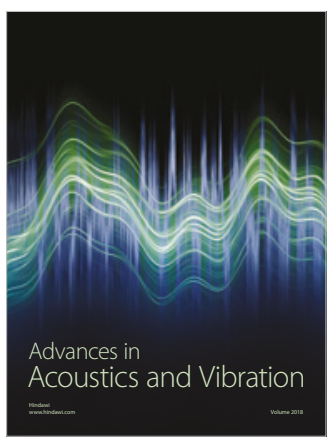

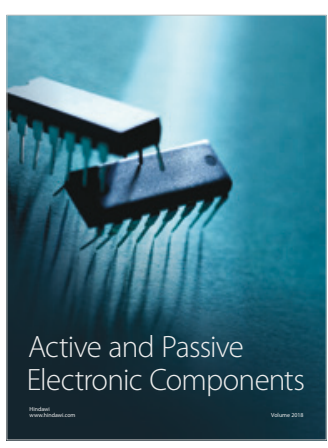
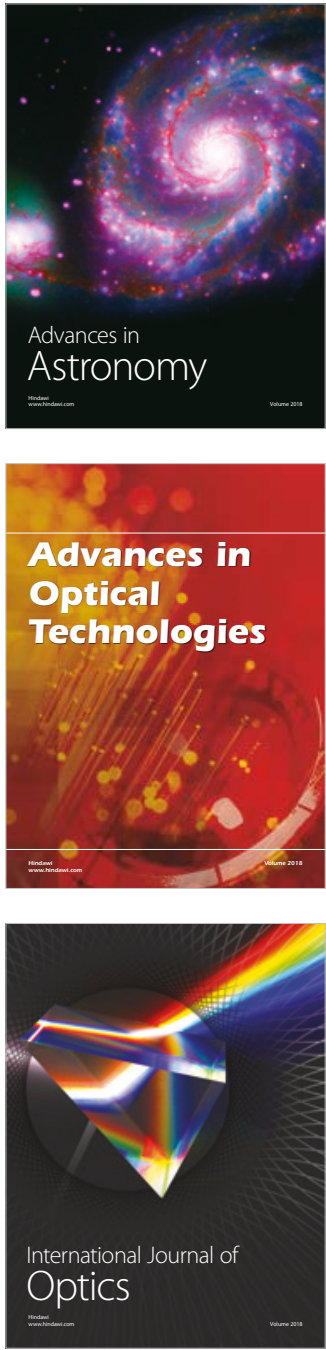